\title{
Segmenting the Endocardial Border of the Left Ventricle in Cardiac Magnetic Resonance Images
}

\author{
H El-Messiry ${ }^{1}$, HA Kestler ${ }^{1,2}$, O Grebe ${ }^{2}$, H Neumann ${ }^{1}$ \\ ${ }^{1}$ Neural Information Processing, University of Ulm, Germany \\ ${ }^{2}$ Medicine II - Cardiology, University Hospital Ulm, Germany
}

\begin{abstract}
The proposed method is composed of two phases: Segmentation phase: uses a bottom-up multi-scale analysis, based mainly on taking the advantage of using the morphological scale-space by decomposing the image into a number of scales of different structure size. As a result of the decomposition, the gray level appearance structures adjacent to the endocardial border are located, and finally an estimated boundary is obtained regardless of those structures. Refinement phase: Asserts prior information about local structure around defined points along the shape boundary. Our approach is able to correctly locate and classify the inner structures in $91 \%$ of the tested $220 \mathrm{MR}$ images from different cases (imaged on 1.5T whole body scanner). The estimated boundary converges correctly to the main endocardial border. In conclusion, this new approach represents a step towards automatic left ventricle segmentation
\end{abstract}

\section{Introduction}

Automated segmentation to find the endocardial boundary of the left heart ventricle from magnetic resonance (MR) images has shown to be a difficult task. One of the major problems related to the detection of the boundary are the shortcomings typical of discrete data, such as sampling artifacts and noise, which may cause the shape boundaries to be indistinct and disconnected. Furthermore, the graylevel appearance of structures inside the ventricular cavities, such as papillary muscles, are often indistinguishable from structures of interest for diagnostic analysis, such as the moving inner heart boundary. Thus, segmentation appears error-prone and often incomplete.

\section{Methods}

\subsection{Method overview}

There exist a number of different approaches that employ different models for the endocardial boundary segmentation. But most of the applied techniques fail in over passing the appearance structures inside the ventricular cavities, which implies error in segmentation. We present a method that combines a bottom-up approach, which gives a good initialization position, since the approach determines the inner cavity and over passes the inner appearance structures inside it. With a high-level approach in one framework, through building a statistical model that describes the variability in border instantiation in terms of prior distribution on deformations of a template.
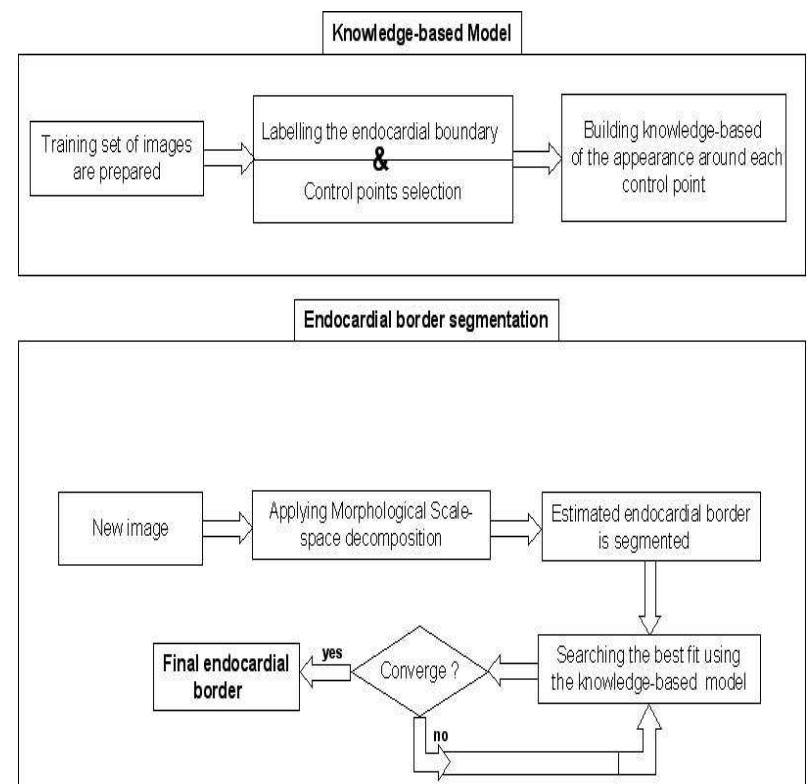

Figure 1. Flowchart illustrating the the combination of the knowledge-based model with a bottom-up model for the endocardial border segmentation.

\subsection{Morphology scale-space decomposition}

A bottom-up model is defined, using morphological scale-space decomposition based on multiscale spatial 
analysis. This method comprises a powerful tool which presents many advantages: the preservation of scalespace causality, the localization of sharp-edges, and the reconstruction of the original image from the scale-space decomposition [1]. In combination with descriptor scale, which defined as the scale that maximizes the response of the morphological filter through the scale-space at each point. The gray level appearance structures adjacent to the endocardial border are located, and finally an estimated boundary is obtained regardless of those structures.

\subsubsection{Morphological scale-space}

Mathematical morphology is a nonlinear analysis of signals, using structuring elements. Two dual operations, erosion and dilation, are the most basic morphological operators. Erosion is shrinking operation while dilation is an expanding one. By combining dilation and erosion two new operations opening and closing can be defined. The Morphological band-pass filter is defined according to [2], by the following formula:

for $n \geq 0$ :

$$
\begin{aligned}
H_{n+1} & =f \\
B_{n} & =\left(H_{n+1} \odot d_{n}\right) \\
H_{n} & =H_{n+1}-B_{n}
\end{aligned}
$$

where $\odot$ is either the opening or the closing operator, $f$ the given image.

The resulting $B_{n}$ represent a morphological decomposition of the image into bands of different structure sizes with light and dark blobs (limited with structure size $d_{n}$ ). $H_{n}$ are intermediate high-pass filtered images. Equation (1) shows a recursive algorithm that alternately high- and lowpass filters the image starting with high-pass filtering at the coarsest scales.

\subsubsection{Closing and Opening descriptor scales}

The Descriptor scale is defined as the scale that maximizes the response of the band-pass morphological filter at each point in the image. This scale gives constant values in a region of constant width, using the definition given by [2], the difference that we defined a descriptor scale for each morphological scale-space obtained from the opening-closing operators separately. According to the equation:

$$
S_{D}=\arg _{S_{k}}\left(\max _{S_{k}=S_{1}, \ldots, S_{n}}\left|\frac{B_{S k}}{S_{k+1}-S_{k}}\right|\right)
$$

\subsubsection{Algorithms}

The algorithm was achieved by linking the descriptor scales, with the scale-space decomposition obtained from the opening and closing morphological operators, in order

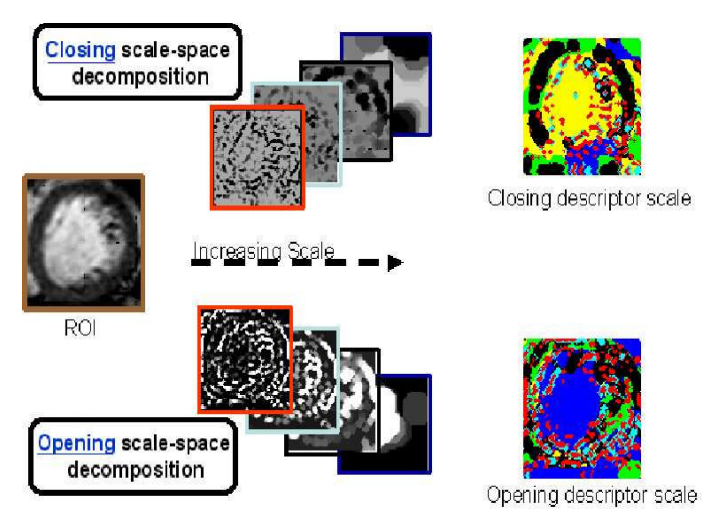

Figure 2. Decomposition of ROI with respect to structure sizes, using the closing and opening morphology operators, and obtaining the maximum response along the scale space.

to distinguish between the inner cavity and the inner graylevel structures inside it, which facilitate the inner boundary segmentation. The algorithm goes as follow:

1. Calculate the morphological band-pass filter based on (1), using a disk as a flat structuring element of increasing logarithmically, obtaining a close scale-space and an open scale-space respectively.

2. Applying (2) for both close and open scale-space, two descriptor scales are obtained.

3. Based on the evaluation of the opening-closing scalespace decomposition and referring to the descriptor scales:

(a) Determine the inner region as follows:

(i) The scale that describes the inner region is selected, according to the opening descriptor scale and refereing to the opening scale-space decomposition.

(ii) Apply a region growing algorithm to detect the region of interest:

- Calculate the center of mass of the scale.

- Select the brightness intensity in 8-connected neighbors to represent the seed point for the region growing.

- Stopping criteria using a defined threshold value.

(b) Determine the inner appearance structures as follows:

(i) Using the closing descriptor scale and refereing to the closing scale-space decomposition. The scale describing the appearance structures is selected.

(ii) Applying gray level histogram with defined threshold, on the selected scale, the appearance structures are located. 4. Combing the information obtained from both the inner region and inner appearance structure procedures, estimated endocardial boundary is obtained.

\subsection{Appearance knowledge-based model}

The contribution in this section is based on refining the fitting of the estimated boundary, obtained by the introduced model in the previous section, since the 


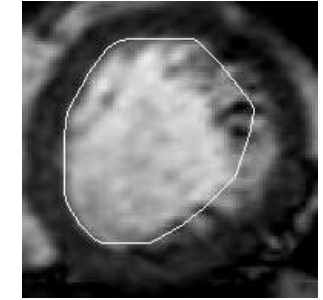

Figure 3. Final estimated boundary.

estimated boundary needs to be enhanced in some locations to obtain the best fit of the boundary to the true endocardial border. The main idea is similar to the searching method proposed by Cootes [3] through the training set a statistical model of the radial gray profiles, normal to the model boundary through each defined control point is build, and during search simply find the points which best match the model.

\subsubsection{Labelling the training set}

For generating a training set, each boundary of the training image is represented by a set of labelled points. We apply a manual procedure for labelling the training set guided by an expert knowledge database (figure 4).

\subsubsection{Control points selection}

From the generated set of labelled points a set of control points are selected, which are separated by equal orientation and located along a certain path using the approach as follows:

1. Calculate the average center of mass for all labelled boundary of the training images (in order to align the labelled points of the training boundaries into a common co-ordinate frame).

2. For each training image do:

(a) Take the average center of mass as the point of origin.

(b) Perform radial decomposition with 15 degree rotational angle, along the labelled points.

(c) Select the intersection point as a control point (figure 4).

The vector describing the $n$ control points of the boundary in the training set is

$$
\mathrm{x}=\left(x_{1}, y_{1}, x_{2}, y_{2}, \ldots, x_{n}, y_{n}\right)^{T}
$$

\subsubsection{Modelling gray appearance knowledge}

We will discuss how we can build the statistical model around each control point as described by Cootes. The main idea is to gather the gray levels information in a region around each control point throughout the training set, concentrating on the gray profiles along perpendicular line passing through the control point in the direction of the selected origin (average center of mass).
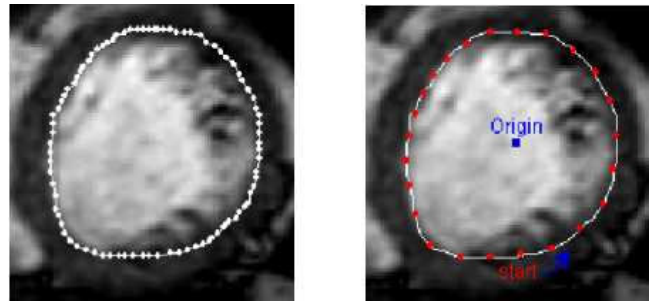

Figure 4. Training image. Left image: labelled endocardial border. Right image: control points selection with 15 degree difference in clockwise direction for the same training image.

For every control point $j$ in the image $i$ of the training set, we extract a gray level profile $\mathbf{g}_{i j}$, of length $n$ pixels, centered around the control point. We do not use the actual gray level profile but its normalized derivative, to reduce the effect of global intensity changes.

The normalized derivative profile is given by

$$
\text { gnorm }_{j}=\frac{d \mathbf{g}_{i j}}{\sum_{k=1}^{n-1}\left|d \mathbf{g}_{i j k}\right|}
$$

Now, we calculate the mean of the normalized derivative profiles of each control point throughout the training set, and we get for control point $j$

$$
\bar{g}_{j}=\frac{1}{N} \sum_{i=1}^{N} \text { gnorm }_{i j}
$$

The covariance matrix of the normalized derivatives is given by

$$
C_{g j}=\frac{1}{N} \sum_{i=1}^{N}\left(\text { gnorm }_{i j}-\bar{g}_{j}\right)\left(\text { gnorm }_{i j}-\bar{g}_{j}\right)^{T}
$$

with this we obtain a statistical model for the gray levels around each control point $j$ represented by $\bar{g}_{j}$ and $C_{g j}$

\subsubsection{Optimizing using gray appearance knowledge}

For a given example, the search process was initialized using the estimated boundary, which obtained from the morphological scale-space decomposition model, the search algorithm is summarized as follows:

1. Compute the control points for the given estimated boundary.

2. for each control point do:

(a) Sample a profile $m$ pixels either side of the current point .

(b) Compute the derivative and normalize of sub-profile $g_{s}$.

(c) Evaluate the error and search the best sub-profile with respect to $g_{s}$ according to the following equation.

$$
\left.f\left(g_{s}\right)=\left(g_{s}-\bar{g}_{j}\right)^{T} C_{g j}^{-1}\left(g_{s}-\bar{g}_{j}\right)\right)
$$




\section{Results and conclusion}

\subsection{Image acquisition and pre-processing}

Images were acquired using a $1.5 \mathrm{~T}$ whole body scanner (Intera CV, Philips Medical Systems) with Master Gradients (slew rate $150 \mathrm{~T} / \mathrm{m} / \mathrm{s}$, amplitude $30 \mathrm{mT} / \mathrm{m}$ ) and a 5-element phased-array cardiac coil. Three short survey scans were performed to define the position and true axis of the left ventricle. Afterwards, wall motion was imaged during breath holding in long and short-axis slices using a steadystate free precession sequence, which provides an excellent demarcation of the endocardium. Cardiac synchronization was achieved by prospective gating. The cine images were recorded with 23 heart phases (23 frames per heart cycle). Each frame of $256 \times 256$ pixels with a slice thickness of $10 \mathrm{~mm}$. For each frame a region of interest was extracted of size $95 \times 95$ pixels.

\subsection{Experimental results and conclusion}

Morphological scale-space decomposition was tested on $220 \mathrm{MR}$ images from different cases. Which is able to correctly locate and classify the inner structures in $91 \%$ of the tested cases. Figure 5 demonstrates using the appearance knowledge search, as the search progresses more suitable adjustments are made. The final convergence (the max control point iteration was 19 iterations) gives a good match to the endocardial boundary. We can see how the algorithm guides the control points in such away that large movements are made for the control points which are far from the best fit position, while small movements for the control points which are near to the optimum fit.

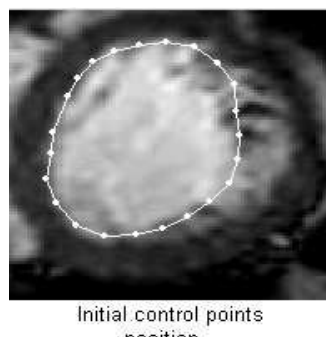
position

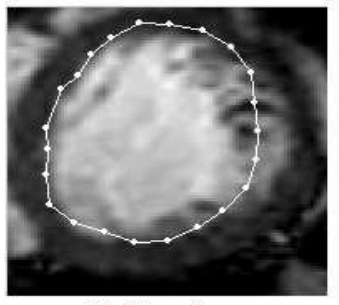

After 7 iteration

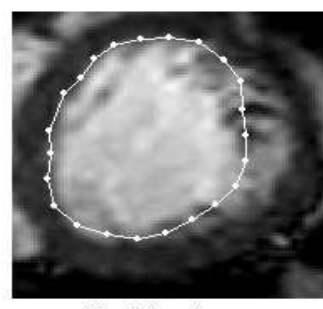

After 3 iteration

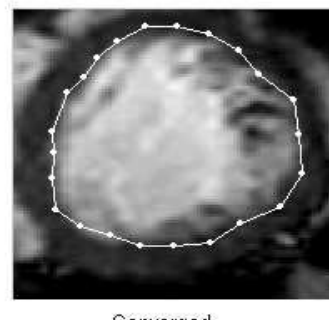

Converged
Figure 5. The final result for the endocardial boundary segmentation using the appearance knowledge search.
The results for the whole model were recorded and compared based on the square error distance (point to point) between the final best fitting given by proposed model and the hand-drawn contour. Figure 6 shows the mean errors with \pm standard deviation for every control point. The maximum control point error within the tested data set was 5 pixels. In conclusion a new combined approach to segmentation of the endocardial boundary of the left ventricle from magnetic resonance images was reported. The developed model gives a new strategy initialization matching approach. It promises to facilitate fully automated quantitative analysis of endocardial boundary in routinely acquired clinical MR images.

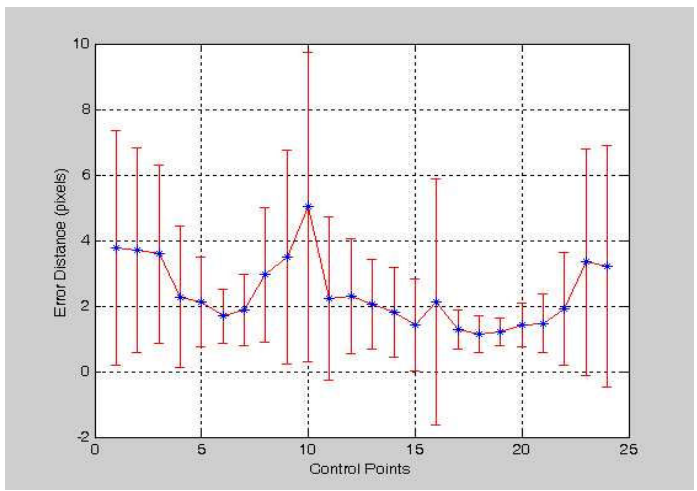

Figure 6. The mean square error distance (y-axis) for each control point (x-axis), with standard error.

\section{Acknowledgements}

H. El-Messiry would like to thank the DAAD for the exchange scholarship PhD program. And Professor Dr. Günther Palm's support is also gratefully acknowledged.

\section{References}

[1] Bangham A, Chardaire P, Ling P. The multiscale morphology decomposition theorem. Mathematical Morphology and Its Application to Image Processing 1994;Proc. of ISMM'94.

[2] Köthe U. Local appropriate scale in morphological scalespace. Computer Vision Proc of 4th European Conference on Computer Vision 1996;1:219-228.

[3] Cootes TF, Hill A, Taylor CJ, Haslam J. The use of active shape models for locating structure in medical images. Images and Vision Computing 1994;12:355-366.

Address for correspondence:

Haythem El-Messiry

Dept. of Neural Information Processing / University Ulm Oberer Eselsberg / D-89069 Ulm / Germany

hmessiry72@yahoo.com 